01

\title{
Столкновения с участием поляризованных щелочных атомов цезия и лития
}

\author{
(C) В.А. Картошкин \\ ФТИ им. А.Ф. Иофрфе, \\ 194021 Санкт-Петербург, Россия \\ e-mail: victor.kart@mail.ioffe.ru
}

Поступила в редакцию 27.12.2019 г.

В окончательной редакции 27.12.2019 г.

Принята к публикации 09.01.2020 г.

Рассчитаны сечения спинового обмена и сдвига частоты магнитного резонанса при столкновении атомов лития и цезия в основном состоянии. Расчет проведен на основании данных по синглетному $\left(X^{1} \Sigma^{+}\right)$и триплетному $\left(a^{3} \Sigma^{+}\right)$потенциалам взаимодействия димера ${ }^{7} \mathrm{Li}^{133} \mathrm{Cs}$. Переход от энергетических к температурным зависимостям действительной и мнимой частей комплексного сечения спинового обмена позволяет получить информацию как об уширении линии магнитного резонанса исследуемых атомов, так и о сдвиге частоты магнитного резонанса при их столкновении.

Ключевые слова: спиновый обмен, поперечные сечения, сдвиги частоты магнитного резонанса.

DOI: $10.21883 /$ OS.2020.04.49197.347-19

\section{Введение}

Исследование столкновительных процессов с участием щелочных атомов вызывает интерес как с экспериментальной, так и с теоретической точек зрения. Щелочные атомы являются рабочими средами при создании приборов квантовой электроники (квантовые магнитометры, стандарты частоты) [1,2]. Кроме того, они широко используются при проведении экспериментов с „холодными атомами“ при температурах ниже $\mathrm{mK}$ [3], в том числе при экспериментах по ультрахолодным столкновениям [4] и химическим реакциям при низких температурах [5]. В частности, димеры LiCs были обнаружены в магнитооптических ловушках [1]. Особый интерес вызывают столкновения с участием поляризованных атомов. Поскольку рассматриваемые щелочные атомы могут быть поляризованы в основном состоянии с помощью резонансного поляризованного оптического излучения, то при их столкновении, кроме упругого рассеяния, возможна передача поляризации партнеру столкновения. При этом спинобменные столкновения приводят к уширению линий магнитного резонанса и сдвигу частоты линий магнитного резонанса [6], а также к образованию высших поляризационных моментов [7] исследуемых атомов. Оба этих процесса влияют на точностные характеристики приборов квантовой электроники. Для учета влияния спин-обменных столкновений на ширины линий и сдвиги частоты магнитного резонанса необходимо знание комплексных сечений спинового обмена сталкивающихся атомов и зависимости этих сечений от температуры.

\section{Синглетный и триплетный потенциалы взаимодействия димера ${ }^{133} \mathrm{Cs}^{7} \mathrm{Li}$}

При столкновении щелочных атомов Cs и Li в основном состоянии образуется молекула LiCs. Поскольку сталкивающиеся атомы в основном состоянии имеют электронные спины, равные $1 / 2$, то образующаяся молекула может находиться в двух состояниях с полными спинами $S$, равными 0 (синглетное состояние $X^{1} \Sigma^{+}$) и 1 (триплетное состояние $a^{3} \Sigma^{+}$). Синглетный и триплетный термы, описывающие взаимодействие атомов Cs и $\mathrm{Li}$ в основном состоянии, были представлены в работе [8]. В этой работе методом фурье-спектроскопии высокого разрешения получены и представлены в виде таблиц данные для синглетного и триплетного потенциалов взаимодействия димера ${ }^{7} \mathrm{Li}^{133} \mathrm{Cs}$.

На рис. 1 приведены потенциалы взаимодействия для синглетного $\left(X^{1} \Sigma^{+}\right)$и триплетного $\left(a^{3} \Sigma^{+}\right)$термов димера ${ }^{133} \mathrm{Cs}^{7} \mathrm{Li}$, построенные в атомной системе единиц. В [8] даются следующие значения параметров для этих потенциалов: синглетный потенциал - энергия диссоциации $D_{e}=5875.455 \mathrm{~cm}^{-1}$ (0.0267627 a.u.), равновесное расстояние $R_{e}=3.66681 \AA \quad\left(6.932 a_{0}\right)$; триплетный потенциал $-D_{e}=309 \mathrm{~cm}^{-1}$ (0.0014075 a.u.), $R_{e}=5.2472 \AA\left(9.919, a_{0}\right)$. Используя эти данные, можно рассчитать комплексные сечения спинового обмена и на их основе определить сдвиги частоты магнитного резонанса щелочных атомов при столкновении. 


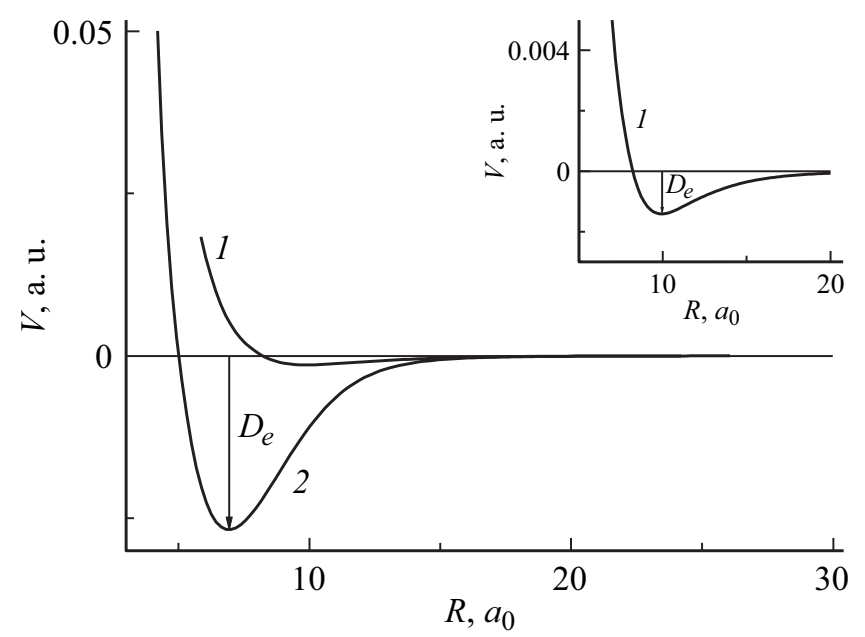

Рис. 1. Потенциалы взаимодействия атомов ${ }^{7} \mathrm{Li}^{133} \mathrm{Cs}$ по данным [8]: $1-$ синглетный $\left(X^{1} \Sigma^{+}\right), 2-$ триплетный $\left(a^{3} \Sigma^{+}\right)$. На вставке - синглетный потенциал в увеличенном масштабе.

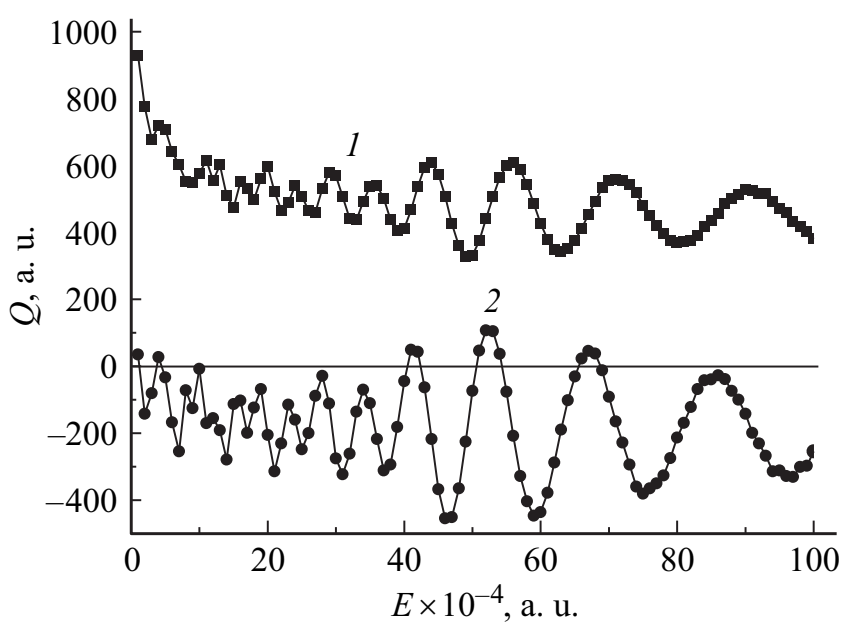

Рис. 2. Зависимость действительной (1) и мнимой (2) частей комплексного сечения спинового обмена от энергии столкновения в системе двух атомов ${ }^{7} \mathrm{Li}^{133} \mathrm{Cs}$ (атомная система единиц).

\section{Расчет комплексных сечений спинового обмена при столкновении атомов цезия и лития}

В процессе столкновения и образования молекулы из двух щелочных атомов, если один из сталкивающихся атомов обладал до момента столкновения электронной поляризацией (полученной, например, при оптической ориентации атомов), то возможна также передача этой поляризации партнеру столкновения. Следует отметить, что в процессе столкновения атомы обмениваются только электронной поляризаций. В результате связи электронного момента атома $\mathbf{S}$ с его ядерным моментом I возникает полный момент $\mathbf{F}$. Таким образом, атом поляризован как по электронному, так и по ядерному спинам.
Перераспределение поляризации между электронными и ядерными моментами было ранее подробно рассмотрено в [9] на примере столкновений метастабильных поляризованных атомов гелия с атомами Rb. Процесс спинового обмена можно условно представить в следующем виде:

$$
A(\uparrow)+B(\downarrow) \rightarrow(A B) \rightarrow A(\downarrow)+B(\uparrow) .
$$

Стрелки указывают направление углового момента атома (в нашем случае - электронного спина). Расчет спинового обмена можно сделать в рамках формализма комплексного сечения [10] аналогично тому, как это было сделано в [11]:

$$
q^{A B}=\stackrel{-A B}{q}+i \stackrel{{ }^{A B}}{q} .
$$

Комплексное сечение спинового обмена имеет следующий вид:

$$
q^{A B}=\frac{\pi}{k_{A B}^{2}} \sum_{l=0}^{\infty}(2 l+1)\left[1-T_{0}^{A B}(l) T_{1}^{A B}(l)^{*}\right] .
$$

Здесь $k_{A B}-$ волновое число, * обозначает комплексное сопряжение, $T$ - матрица рассеяния на синглетном или триплетном термах. Матрица рассеяния $T$, в свою очередь, может быть представлена через фазы рассеяния $\left(\delta_{S}^{A B}(l)\right)$ в канале с полным спином $S$ следующим обра3ом:

$$
T_{S}^{A B}(l)=\exp \left(2 i \delta_{S}^{A B}(l)\right),
$$

где $l$ - орбитальное квантовое число.

Принимая во внимание (3) и (4), действительную и мнимую части комплексного сечения можно выразить через фазы рассеяния на соответствующих термах:

$$
\stackrel{-}{q}^{A B}=\frac{\pi}{k_{A B}^{2}} \sum_{l=0}^{\infty}(2 l+1) \sin ^{2}\left[\sigma_{1}^{A B}(l)-\sigma_{0}^{A B}(l)\right],
$$

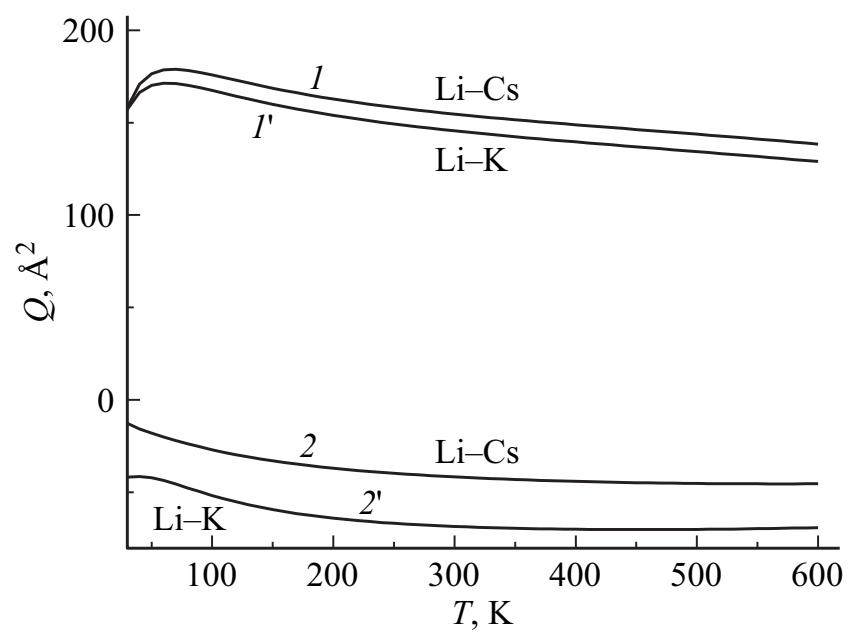

Рис. 3. Зависимость действительной $\left(1,1^{\prime}\right)$ и мнимой $\left(2,2^{\prime}\right)$ частей комплексного сечения спинового обмена от температуры при столкновении атомов ${ }^{7} \mathrm{Li}$ со щелочными атомами Cs и $\mathrm{K}[13]$. 


$$
\stackrel{={ }^{A B}}{q}=\frac{\pi}{k_{A B}^{2}} \sum_{l=0}^{\infty}(2 l+1) \sin 2\left[\delta_{1}^{A B}(l)-\delta_{0}^{A B}(l)\right] .
$$

Зная синглетный и триплетный потенциалы взаимодействия молекулы LiCs, можно рассчитать фазы рассеяния на синглетном и триплетном термах и определить интересующие нас сечения. Фазы рассеяния рассчитывались в квазиклассическом приближении в интервале энергий столкновения $10^{-4}-10^{-2}$ a.u. с использованием приближения Джефриса, модифицированного Ламбером [12]:

$$
\delta_{s}=\int_{R_{0}}^{\infty} F_{1}(R) d R-\int_{R_{0}^{\prime}}^{\infty} F_{0}(R) d R
$$

где

$$
\begin{gathered}
F_{1}^{S}(R)=\left[2 \mu\left(E-V_{S}(R)-\frac{(l+1 / 2)^{2}}{2 \mu R^{2}}\right)\right], \\
S=s, t, q, \\
F_{0}(R)=\left[2 \mu E-\frac{(l+1 / 2)^{2}}{R^{2}}\right] .
\end{gathered}
$$

Здесь $E-$ кинетическая энергия, $R_{0}$ и $R_{0}^{\prime}-$ корни уравнений $F_{1}^{S}(R)=0$ и $F_{0}(R)=0$ (для $F_{1}^{S}$ берется больший корень), $V_{S}(R)$ - потенциал взаимодействия, соответствующий полному спину $S, \mu-$ приведенная масса сталкивающихся частиц, $l-$ орбитальное квантовое число. На рис. 2 приведены полученные в результате расчета зависимости действительной $\bar{q}$ и мнимой $\overline{\bar{q}}$ частей комплексного сечения от энергии столкновения. Как видно из рисунка, сечение сдвига частоты LiCs находится в основном в области отрицательных значений.

\section{Заключение}

На рис. 3 приведены усредненные по скоростям зависимости действительной и мнимой частей комплексного сечения спинового обмена в интервале температур 30-600 К. Как видно из рисунка, усредненное сечение сдвига для LiCs имеет отрицательное значение во всем интервале рассматриваемых температур. Для сравнения на рисунке приведены зависимости аналогичных сечений для $\mathrm{LiK}$.

Таким образом, полученные в настоящей работе температурные зависимости действительной и мнимой частей комплексного сечения спинового обмена позволяют рассчитать вклад спин-обменного процесса в ширину и сдвиг линии магнитного резонанса при столкновении между поляризованными атомами лития и цезия. Однако $[14,15]$ поскольку наряду со столкновениями между атомами Li и Cs в камере поглощения происходят столкновения и между одинаковыми атомами $(\mathrm{Li}-\mathrm{Li}, \mathrm{Cs}-\mathrm{Cs})$, то для проведения расчета необходимо также знание комплексных сечений спинового обмена, описывающих столкновения одинаковых атомов.

\section{Конфрликт интересов}

Автор заявляет, что у него нет конфликта интересов.

\section{Список литературы}

[1] Александров Е.Б., Вершовский А.К // УФН. 2009. Т. 179. № 6. C. 605-637; Aleksandrov E.B., Vershovskii A.K. // Phys.Usp. 2009. V. 52. N 6. P. 573.

[2] Риле Ф. Стандарты частоты. Принципы и приложения. М.: Физматлит, 2009. 512 с.

[3] Kraft S.D., Staanum P., Lange J. et al. // J. Phys. B. 2009. V. 39. P. S993.

[4] Krems R.V. // Phys. Rev. Lett. 2006. V. 96. P. 123202.

[5] Bodo E., Gianturco F., Dalgarno A. // J. Chem. Phys. 2002. V. 116. P. 9222.

[6] Happer W., Jau Y.-Y., Walker T. // Optically Pumped Atoms. WILEY-VCH. 2010. P. 246.

[7] Дмитриев С.П., Доватор Н.А., Картошкин В.А., Окуневич А.И. // Опт. и спектр. 1994. Т. 77. № 5. C. 712-713; Dmitriev S.P., Dovator N.A., Kartoshkin V.A., Okunevich A.I. // Opt. Spectrsc. 1994. V. 77. N 5. P. 712.

[8] Staanum P., Pashov A., Knockel H., Tiemann E. // Phys. Rev. A. 75. 2007. P. 042513.

[9] Дмитриев С.П, Доватор Н.А., Картошкин В. А., Клементьев Г.В. // Опт. и спектр. 2016. Т. 121. № 5. C. 689-694; Dmitriev S.P., Dovator N.A., Kartoshkin V.A., Klementiev G.V. // Opt. Spectrosc. 2016. V. 121. N 5. P. 649-654.

[10] Happer W. // Rev. Mod. Phys. 1972. V. 44. P. 169-249.

[11] Картошкин В.А. // Опт. и спектр. 2010. Т. 109. № 5. C. 733-738; Kartoshkin V.A. // Opt. Spectrosc. 2010. V. 109. N 5. P. 674-679.

[12] Мотm Н., Месси Г. Теория атомных столкновений. М.: Мир, 1969. 756 с.

[13] Картошкин В.A. // Опт. и спектр. 2019. Т. 127. № 4. C. 639-641; Kartoshkin V.A. // Opt. Spectrosc. 2019. V. 127. N 4. P. 691-693.

[14] Окуневич А.И. //Опт. и спектр.1995. Т. 79. № 5. С. 718.

[15] Картошкин В.A. // Опт. и спектр. 2010. Т. 108. № 6. C. 914-917; Kartoshkin V.A. // Opt. Spectrosc. 2010. V. 108. N 6. P. 866. 